\title{
Blocking Mammalian Target of Rapamycin (mTOR) Alleviates Neuropathic Pain Induced by Chemotherapeutic Bortezo.
}

\author{
Zongsheng Duan ${ }^{\mathrm{a}}$ Jing Li ${ }^{\mathrm{b}} \quad$ Xiaochuan Pang ${ }^{\mathrm{c}}$ Hushan Wang ${ }^{\mathrm{a}}$
}

aDepartment of Anesthesiology, The First Hospital of Jilin University, Changch Radiology, The First Hospital of Jilin University, Changchun, Jilin, co Labora of Jilin University, Changchun, Jilin, dDepartment of Anesthesiolo Hospital of Jilin University, China

\section{Key Words}

Mammalian target of rapamycin • Neuropathic pamycin $•$ Signal pathway

\section{Abstract \\ Background/Aims: Bortezomib (BTZ) the treatment of cancer. However, o} painful peripheral neuropathy durin painful symptoms induced $b$ neuropathic pain remain larg effects of blocking mamr "an hypersensitivity evoked $r$ MTOR in regulating BTZ to determine mechanica, ELISA were user' signals, and th Systemic i compar p'edinte ctio th co \section{(f)} \Z and are

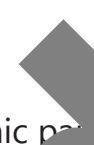
uced neuropathic pain. Methods: Behavioral test was performed since the underlying mechanisms leading to as a chemotherapeutic agent for o. signiticant limiting complications of BTZ is py. Drugs preventing and/or treating the clear. me purposes of this study were to examine 1 ) the $f$ rapamycin (mTOR) on mechanical pain and cold underlying mechanisms responsible for the role of cold sensitivity in a rat model. Western blot analysis and ine expression of mTOR and phosphatidylinositide 3-kinase (p-PI3K) ybstance P and calcitonin gene-related peptide (CGRP). Results: significantly increased mechanical pain and cold sensitivity as animals $(P<0.05$ vs. control rats). The expression of $p-m T O R$, mTORsphorylation of p70 ribosomal S6 protein kinase 1 (p-S6K1), 4E-binding protein L-BP_ yell as $p$-PI3K was amplified in the dorsal horn of spinal cord of BTZ rats as ed wit control rats. Blocking mTOR by intrathecal infusion of rapamycin attenuated mech. Jain and cold hypersensitivity. Blocking PI3K signal also attenuated activities of $\mathrm{OR}$, wrich was accompanied with decreasing neuropathic pain. Inhibition of either mTOR
O data for the first time revealed specific signaling pathways leading to BTZ-induced eripheral neuropathic pain, including the activation of mTOR and PI3K. Inhibition of these signal pathways alleviates pain. Targeting one or more of these signaling molecules may present new opportunities for treatment and management of peripheral painful neuropathy observed during chemotherapeutic application of BTZ.

Zongsheng Duan and Jing Li contributed equally to this work. 


\section{Cellular Physiology \begin{tabular}{l|l|l} 
and Biochemistry $\begin{array}{l}\text { DOI: 10.1159/000491662 } \\
\text { Published } 2018 \text { The Author(s). Published by S. Karger AG, Basel } \\
\text { www.karger.com/cpb }\end{array}$ \\
\hline
\end{tabular} \\ Duan et al.: mTOR and Peripheral Neuropathic Pain}

\section{Introduction}

Bortezomib (BTZ), a reversible inhibitor of the proteasome complex, is used as a chemotherapeutic agent for the treatment of cancer such as multiple myeloma $[1,2]$. However, the prior studies have demonstrated that dose-limiting painful periphera neuropathy is one of the significant adverse events observed during BTZ therapy [. Generally, treatment options for these abnormal sensations have been restricted, partly due to a poor understanding of the underlying mechanisms leading to neuropathic pain induc by BTZ. Drugs preventing and/or treating the painful symptoms evoked by BTZ therapy lacking. Therefore, it is significant to determine molecular mediators of BTZ-in ed pa neuropathy to make therapeutic strategies and have management of chemotrer sut patients with multiple myeloma.

Mammalian target of rapamycin (mTOR) is a serine threonine proteir two distinct mTOR forms of protein complexes, mTOR complex 1 (mTORC1 general, mTORC1 is composed of raptor, mLST8 and mTOR, and is know ga of most proteins by phosphorylation of specific downstream ef s inclu ribosomal S6 protein kinase (p70 S6Ks) and 4E-BPs [4].

Activation of mTOR, in particular, mTORC1 that is $\mathrm{p}$ to promotion of the phosphorylation of downstream eff further governs mRNA translation [4]. The mTORC1 is we the regulation of protein synthesis and growth and evidence plays a role in modulating long-term neuronak icity $[5,6]$.

sitive amycin, leads p $>$ S6K1 and this its critical roles in orts the notion that mTOR 4E-BP1 are presented in the spinal cord dorsa and con 4 bute to transmission and modulation of pain [6-8]. i.e., intrathecal admin f rapamycin, an inhibitor of mTOR, produces antinociception in models of inflan into the spinal cord attenuates formalin-indu. horn_ENREF_6 [11]. Note that rapamy with downregulated mTOR, S6K1 an its downstream signals are ac "va"ed development of spinal pain $s$

The superficial dorsal $h$

tion 2]. Local perfusion of rapamycin onal hyperexcitability in the dorsal E 12$]$. These findings indicate that mTOR and stent pain conditions and contribute to the

he first synaptic site from peripheral afferent nerves to the central nervous syst 13, 1 plays an important role in modulating pain [15, 16]. Especially, the sup cial dorsar horn at the lumbar levels (i.e., L5 to L6) is the first synaptic site receiving $\mathrm{p}$ rom the hind paw. Thus, in this study we determined the role played by $\mathrm{m}^{\mathrm{T}}$ th of lumbar superficial dorsal horn in regulating mechanical hyperalgesia a cersensitivity in rats with systemic administration of BTZ. We hypothesizod trat in egulates the protein expression of mTOR signal pathways in the superficia rsa resulting in mechanical pain and cold hypersensitivity. Moreover, blockik nal mon by intrathecal injection of rapamycin attenuates the amplified
ain response evoked by BTZ. We also hypothesized that BTZ amplifies sion cosphatidylinositide 3-kinase (PI3K), an upstream signal of mTOR [4]. We S Ye that blocking spinal PI3K would attenuate activities of mTOR pathway thereby din reduction in neuropathic pain.

Moreover, the releases of neurotransmitters such as substance P and calcitonin genere d peptide (CGRP) [17-19], within the dorsal horn play a key role in regulating pain ponses [16]. Numerous neurotransmitters and related receptors in the dorsal horn have been targets to understanding of the underlying mechanisms responsible for pain $[15,16]$. Accordingly, we further hypothesized that blocking mTOR and PI3K pathways attenuates the amplified levels of substance P and CGRP in the superficial dorsal horn of BTZ rats.

\section{Materials and Methods}

Animal model of neuropathic pain

All animal protocols were in accordance with the guidelines of the International Association for the Study of Pain and approved by the Institutional Animal Care and Use Committee of Jilin University. Adult

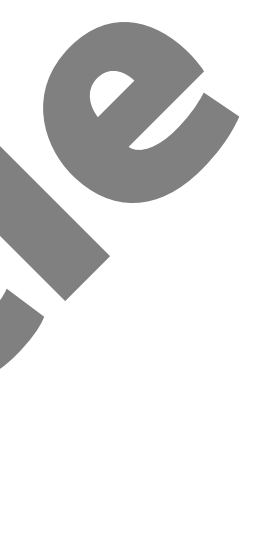




\section{Cellular Physiology Cell Physiol Biochem 2018;48:54-62 and Biochemistry Published onlıne: July $11,2018 \quad \begin{aligned} & \text { DOI: 10.1159/000491662 } 2018 \text { The Author(s). Published by S. Karger AG, Basel } \\ & \text { www.karger.com/cpb }\end{aligned}$ \\ Duan et al.: mTOR and Peripheral Neuropathic Pain}

male Sprague-Dawley (200-250 g) were housed in individual cages with free access to food and water and were kept in a temperature-controlled room $\left(25^{\circ} \mathrm{C}\right)$ on a $12 / 12 \mathrm{~h}$ light/dark cycle. BTZ $(0.4 \mathrm{mg} / \mathrm{kg}$ body weight; Haoran BioTech Co., Shanghai, China) was administrated intraperitoneally (i.p.) once daily for 5 consecutive days. Control animals received an equivalent volume of saline.

\section{Intrathecal catheter for administration of drugs}

One end of polyethylene-10 tubing was inserted intrathecally through an incision in the cisternal membrane and advanced 7-9 cm caudal until the tip of the catheter was positioned at the lumbar sp level (L5 to L6). The other end of the intrathecal tubing was sutured to the musculature and skin ? incision site and externalized to the back of the rat. Animals were allowed to recover and the animal care facility where they were housed individually. Five days were allowed before the were performed.

\section{Behavioral test}

To quantify the mechanical sensitivity of the hindpaw, rats were placed in indi allowed to acclimate for $>30 \mathrm{~min}$. Mechanical paw withdrawal threshold (PWT) to the stimulation of von Frey filaments was determined. A series of crlibrated vo from 0.5 to $18.0 \mathrm{~g}$ ) were applied perpendicularly to the plantar surf hindpav. a sufficient force to bend the filaments for 60s or until paw withdrew. In the presence force was applied. In the absence of a response, the filament of nex vas applied. To avoid injury during tests, the cutoff strength of the von Frey f*lament was 1 re tactile stimulus producing a $50 \%$ likelihood of withdrawal was determined using - 20 ]. Each trial was repeated 2 times at $\sim 2$ min intervals. The mean value was used as t. produced withdrawal response.

Thermal Place Preference System (Coulburn In preference test in order to assess a cold avoidance be by a plastic enclosure. The first plate was kept at nt kept at cold temperature $\left(12^{\circ} \mathrm{C}\right)$. The test $y$ During the session, the rats were left free to entire session was recorded using in inare behavior. To better control behav the arkness and each session lasted 3 minutes. was used to perform the thermal place or. Ty hecting metal plates were surrounded erature $\left(25^{\circ} \mathrm{C}\right)$ and the second plate was olf th plates. The time spent on the cold plate during the repeatedly placed on the apparatus with both held at room temperature $\left(25^{\circ} \mathrm{C}\right)$ minutes 2 days before the beginning of the experiment. Note that rats spent an equal amount of on ea under these conditions, suggesting that animals showed no place preference. To avoid l ing or any prace preference unrelated to cold, the temperature of the plates was inverted between two c ssions. Two trials were made and data were averaged. Note that all behavioral tests we orm find fashion.

Experiment or

Follow coll on baseline data, a Hamilton microsyringe $(250 \mu \mathrm{L})$ was connected to the intrathec Ing to $100 \mu \mathrm{l}$ of dimethyl sulfoxide (DMSO) as vehicle control, antagonists to mTOR

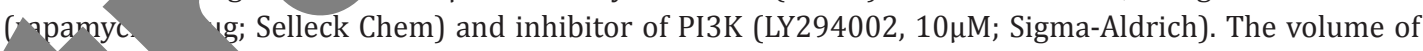
rapa. and LY294002 was $100 \mu \mathrm{l}$. An infusion pump was used to deliver vehicle and drugs and was su 0 constantly over a period of $60 \mathrm{~min}$. Note that a prior study [12] using the same approach to in $\quad$ infuse rapamycin $(1,5$ and $10 \mu \mathrm{g})$ and $\mathrm{LY} 294002(1,5$ and $10 \mu \mathrm{M})$. It was shown that $10 \mu \mathrm{g}$ paminun and $10 \mu \mathrm{M}$ of LY294002 were effective to attenuate mechanical and thermal hyperalgesia. We ad those doses accordingly in our current study.

sixty minutes after intrathecal infusion, mechanical and cold sensitivity of the hindpaws were again ctermined. Thus, six groups were included in this study as: saline rats + vehicle infusion $(n=10)$; BTZ rats + vehicle infusion $(\mathrm{n}=15)$; saline rats + rapamycin $(\mathrm{n}=12)$; BTZ rats + rapamycin $(\mathrm{n}=16)$; saline rats+ LY294002 (n=8); and BTZ rats + LY294002 ( $\mathrm{n}=12)$.

In a subset of experiments, in order to examine the effects of blocking mTOR and PI3K on substance $P$ and CGRP vehicle $(n=12)$, rapamycin $(10 \mu \mathrm{g}, \mathrm{n}=15)$ and LY294002 $(10 \mu \mathrm{M}, \mathrm{n}=15)$ were intrathecally given using an infusion pump, respectively. The pump was set to constantly deliver vehicle or the drugs over a period of $60 \mathrm{~min}$. At the end of infusion, rats were euthanized by sodium pentobarbital (120 mg/kg, i.p.) and the superficial dorsal horn tissues (L4-L6) were obtained under an anatomical microscope for Western Blot and ELISA experiments.

\section{KARGER}




\section{Cellular Physiology Cell Physiol Biochem 2018;48:54-62 and Biochemistry Published online: July 11, $2018 \quad \begin{aligned} & \text { DOI: 10.1159/000491662 } 2018 \text { The Author(s). Published by S. Karger AG, Basel } \\ & \text { www.karger.com/cpb }\end{aligned}$ \\ Duan et al.: mTOR and Peripheral Neuropathic Pain}

Western blot analysis

Total protein of the superficial dorsal horn tissues was extracted by homogenizing dorsal horn sample in ice-cold immunoprecipitation assay buffer. The lysates were centrifuged and the supernatants were collected for measurements of protein concentrations. After being denatured by heating at $95^{\circ} \mathrm{C}$ for 5 min in buffer, the supernatant samples containing $20 \mu \mathrm{g}$ of protein were loaded onto 4-20\% Mini-PROTE TGX gels and electrically transferred to a polyvinylidene fluoride membrane. The membrane was blocked $5 \%$ nonfat milk in $0.1 \%$ Tween-TBS buffer and was incubated overnight with respective primary antibody, including: rabbit anti-p-mTOR /p-S6K1/p-4E-BP1 antibodies (1:200); rabbit anti-mTOR/S6K1/4E-r antibodies (1:200); rabbit anti-p-PI3K p85 (1:250). Next, the membranes were washed and incu with an alkaline phosphatase conjugated anti-rabbit secondary antibody (1:1000). Al antibodies were purchased from the Abcam Co. and/or Cayman Chem Co; and goat anti-rabk antibody was purchased from Santa Cruz Biotech. The immunoreactive proteins were chemiluminescence. The bands recognized by the primary antibody were visualized. membrane onto an x-ray film. The membrane was stripped and incubated with ar show equal loading of the protein. Then, the film was scanned and the optical d analyzed using the Scion Image software (NIH, USA), and values for densities or actin band densities from the same lane were examined. Each of the $y$ was then sample.

\section{ELISA measurements}

To examine the levels of substance $\mathrm{P}$ and $\mathrm{CGR}^{\mathrm{r}}$ the superfic orsal horn of the spinal cord, ELISA methods were employed. Substance P was mo d using sub ce P ELISA kit following the manufacturer's instructions (Abcam Co., Cambridge, $\mathrm{M}^{\wedge} \quad{ }^{\mathrm{q}} \mathrm{V}$, the diluted tissue supernatant $(100 \mu \mathrm{l})$ was placed in a 96-well goat anti-mouse IgG-coated an bated for 2 hours. After incubation, the plate was washed using the provided washing buffer, he co vas developed by adding PNPP (200 $\mu$ l) substrate after $45 \mathrm{~min}$ and determined by an Fula with a wavelength of $575 \mathrm{~nm}$. The amount of substance P was calculated by using a su all tanaard curve. In the similar way, the CGRP content of the samples ( $100 \mu \mathrm{l}$ supernatant) was de Co.). Briefly, the diluted sample well plate incubated with pre-coated anti-rat IgG antibody overnight, washed and d ed, anaryantified [21].

\section{Statistical analysis}

All data were analyzed and post-infusion were comp deviation of mear anaryus, differences were considered significant at $P<0.05$. All statistical analyses were p rr ing SPSS for Windows version 13.0 (SPSS Inc., Chicago, USA).
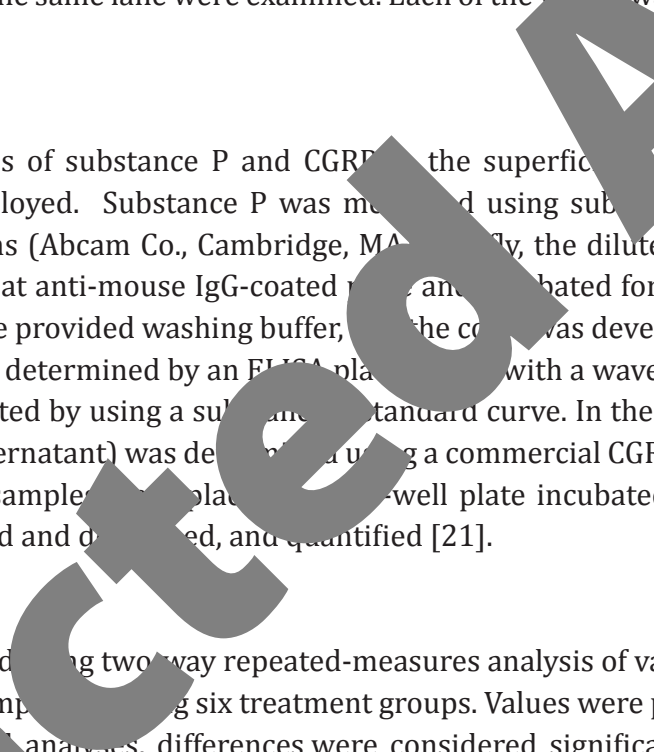

\section{sects os locking mTOR and PI3K on mechanical and cold sensitivity obtained all the baseline data of mechanical and cold sensitivity, control vehicle,} mycn and LY294002 were intrathecally infused. Fig. 1 (top panel) showed that BTZ ased PWT in rats with vehicle infusion $(P<0.05$ vs. saline control rats; $n=15)$ compared saline control rats $(n=10)$. As rapamycin $(n=16)$ and LY294002 $(n=12)$ were infused, a in PWT evoked by BTZ was blunted as compared with PWT in BTZ rat with vehicle infusion $(P<0.05$, vehicle vS. rapamycin/ LY294002 in BTZ rats). Note that there was a smaller increase of PWT observed in saline control rats after infusion either rapamycin $(n=12)$ or LY294002 (n=8), suggesting that blocking mTOR and PI3K had less effects on mechanical sensitivity in rats under healthy conditions.

Fig. 1 (bottom panels) further showed that BTZ decreased \% time spent on the cold plate. As rapamycin and LY294002 were intrathecally infused, the shortened time spent on the cold plate evoked by BTZ was largely recovered. Similarly, a smaller increase of \% time spent on the cold plate observed in saline control rats after infusion either rapamycin or 


\section{Cellular Physiology Cell Physiol Biochem 2018;48:54-62 \begin{tabular}{l|l} 
DOI: 10.1159/000491662 & Ond Biochemistry \\
Published online: July II, 2018 & $\begin{array}{l}\text { 2018 The Author(s). Published by S. Karger AG, Basel } \\
\text { www.karger.com/cpb }\end{array}$
\end{tabular} \\ Duan et al.: mTOR and Peripheral Neuropathic Pain}

LY294002, suggesting that blocking mTOR and PI3K had less effects on cold sensitivity in rats under healthy conditions.

\section{Expression of mTOR signal pathway}

Fig. 2A showed the protein expression of p-mTOR, p-S6K1 and p-4E-BP1 as well mTOR, S6K1 and 4E-BP1 in saline control rats and BTZ rats. BTZ significantly increased th protein levels of p-mTOR and mTOR-mediated p-S6K1 and p-4E-BP1 in the superficial dorsal horn as compared with control rats. Optical density for p-mTOR, p-S6K1 and p-4E-BP1 $1.06 \pm 0.16 ; 1.05 \pm 0.16 ; 1.03 \pm 0.15$ in control rats, and $1.78 \pm 0.26 ; 1.67 \pm 0.25 ; 163 \pm 0.2$ BTZ rats $(P<0.05 v s$. control rats, $\mathrm{n}=6-10$ in each group). Note that total pro i $\mathrm{m}$ S6K1 and 4E-BP1 levels was not significantly increased in BTZ rats. Furtherm demonstrated that the ratio of p-mTOR, p-S6K1 and p-4E-BP1 levels mTOR, S6K1 and 4E-BP1 levels was significantly increased in BTZ rats.

In addition, Fig. 3A showed that expression of PI3K was upregul compared with control rats ( $\mathrm{n}=6$ in each group). We further examined $t$ r PI3K on expression of p-mTOR and p-S6K1. Fig. 3B demonstrated tha of p-mTOR and p-S6K1 was significantly increased in BT7 $\quad(n=6-10 \quad$ compared with saline control rats (n=6-10). When LY294002 was infused inal ca of BTZ rat, the

Fig. 1. Effects of blocking mTOR and PI3K pathways on BTZ-evoked mechanical and cold hypersensitivity. Top panel: BTZ decreased PWT and intrathecal administration of rapamycin and LY294002 blunted the effects of BTZ. Bottom panel: BTZ decreased \% time spent on cold plate. Rapamycin and LY294002 largely recovered the shortened time spent plate induced by BTZ. ${ }^{*} \mathrm{P}<0.05$ vs. their $\mathrm{r}$ pre-infusion; and ${ }^{*} \mathrm{P}<0.05$ vs. prst-infus other groups. \# $\mathrm{P}<0.05$, indicate pre-infusion in respective groups is shown in the Fig. and they y panel. Sal: saline; Veh: vehic rapamycin; and LY: LY2940C $\longrightarrow$ (
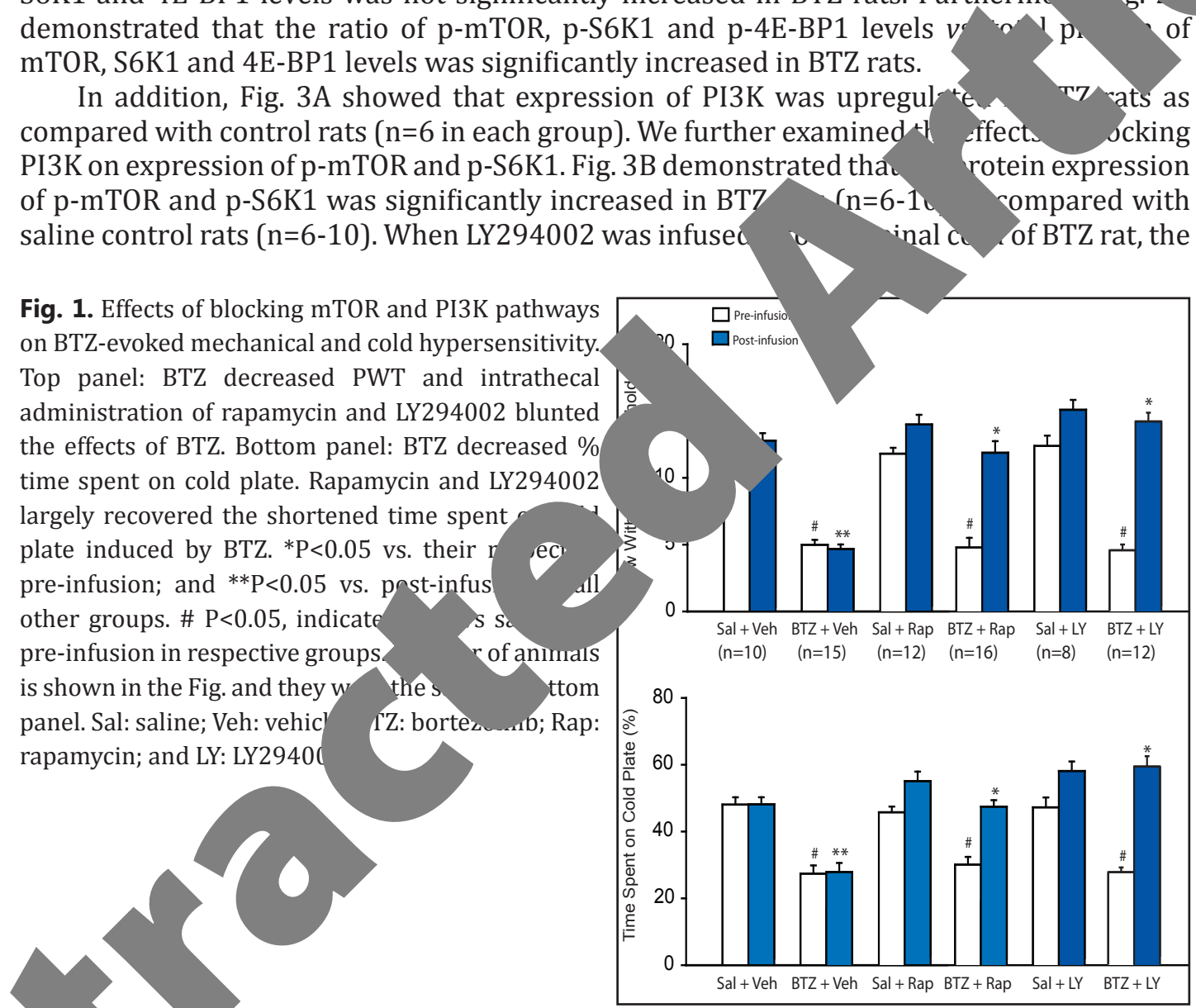

Fig. Ssion of mTOR pathways in control rats

B' A, Top panel: typical bands and bottom rel: averaged data, showing that p-mTOR, p-S6K1 an 4E-BP1 in the dorsal horn of the spinal cord were egulated in $\mathrm{BTZ}$ rats. ${ }^{*} \mathrm{P}<0.05$ vs. control rats $(\mathrm{n}=6$ 10 in each group). There were insignificant differences in total protein expression of mTOR, S6K1 and 4E-BP1 in control rats and BTZ rats $(\mathrm{P}>0.05 . \mathrm{n}=6-10$ in each group). B, The ratio of p-mTOR, p-S6K1 and p-4E-BP1 levels vs. total protein of mTOR, S6K1 and 4E-BP1 levels was significantly increased in BTZ rats. ${ }^{*} \mathrm{P}<0.05$ vs. control rats ( $\mathrm{n}=6-10$ in each group).

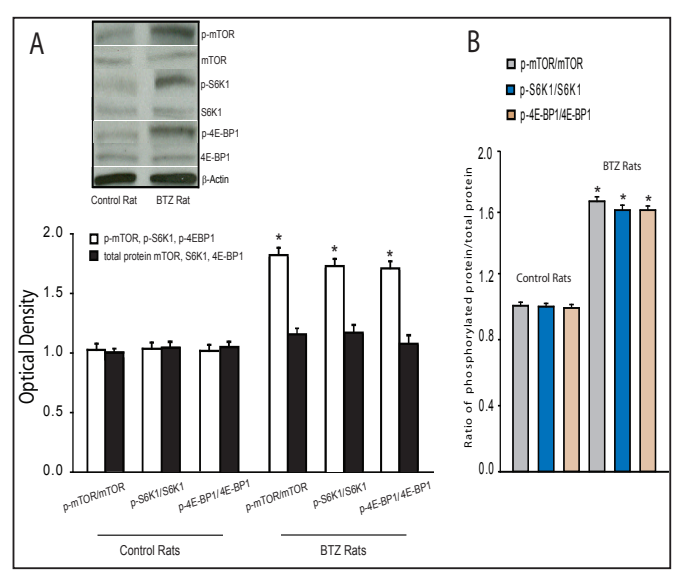


amplified activities of p-mTOR and p-S6K1 evoked by BTZ were significantly attenuated (n=610 in each group). Nonetheless, total protein of mTOR and S6K1 levels was not significantly altered by LY294002 in BTZ rats. Fig. 3C demonstrated that the ratio of p-mTOR and p-S6K1 levels vs. total protein of mTOR and S6K1 levels was significantly increased in BTZ rats and this was inhibited after infusion of LY294002.

\section{Levels of substance P and CGRP}

In additional experiments, we examined the effects of BTZ on the levels of substance $\mathrm{P}$ and CGRP in the superficial dorsal horn of the spinal cord. Fig. 4 showed that substance $P$ and CGRP were significantly increased in BTZ rats with vehicle infusion $(n=12)$ as compared with control rats $(n=10)$. Furthermore, blocking individual mTOR and PI3K signaling pathways by intrathecal infusion of rapamycin and LY294002 $(n=15$ in each group) significantly attenuated amplifications in substance $P$ and CGRP evoked by BTZ. It is noted that LY294002 had a greater inhibitory effect on substance $P$ and CGRP than rapamycin did.

\section{Discussion}

In this report, $\mathrm{w}$ that BTZ upregulated its downstream ciona. dorsal horn Inmuvition Fig.<smiles>[CH2][IH]</smiles><smiles>C1CCCCC1</smiles>

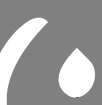
and $\mathrm{PI}$ hways on the levels of ce G GRP. BTZ significantly ed sub ce $P$ and CGRP as com th controls, and blocking TOR PI3K by rapamycin and 94002 significantly attenuated the en cement in substance P and CGRP red by BTZ. Note that LY294002 had a greater inhibitory effect on substance $P$ and CGRP than rapamycin did. ${ }^{*} \mathrm{P}<0.05$, indicated BTZ rats with vehicle infusion $(n=12)$ vs. control rats $(n=10)$ and BTZ rats with rapamycin $(n=15)$ and LY294002 ( $\mathrm{n}=15)$ infusion. \# $\mathrm{P}<0.05$, indicated BTZ rats with rapamycin vs. BTZ rats with LY294002.

\section{KARGER}

Fig. 3. Effects of block $3 \mathrm{~K}$ on mTOR expression. A, Top par vical bands bottom panel: averaged data, showing ex ion of upr alated p- PI3K in STZ rats as compare trol rats ( $\mathrm{n}=6$ in each group). ${ }^{*} \mathrm{P}<0.05$, control TZ. 2: typical bands and averaged data: p-mTOR Nas amplified in the dorsal horn of BTZ eatment as compared with saline control ats cking PI3K signal pathway by intrathecal infusion 292 attenuated increases in p-mTOR and p-S6K1 ats. $* \mathrm{P}<0.05$ vs. control animals and BTZ animals infused with LY294002 ( $\mathrm{n}=6-10$ in each group). C, The ratio p-mTOR and p-S6K1 levels vs. total protein of mTOR and s6K1 levels was significantly increased in BTZ rats. This was attenuated by infusion of LY294002. $*$ P $<0.05$ vs. control rats and BTZ animals infused with LY294002 ( $\mathrm{n}=6-10$ in each group).

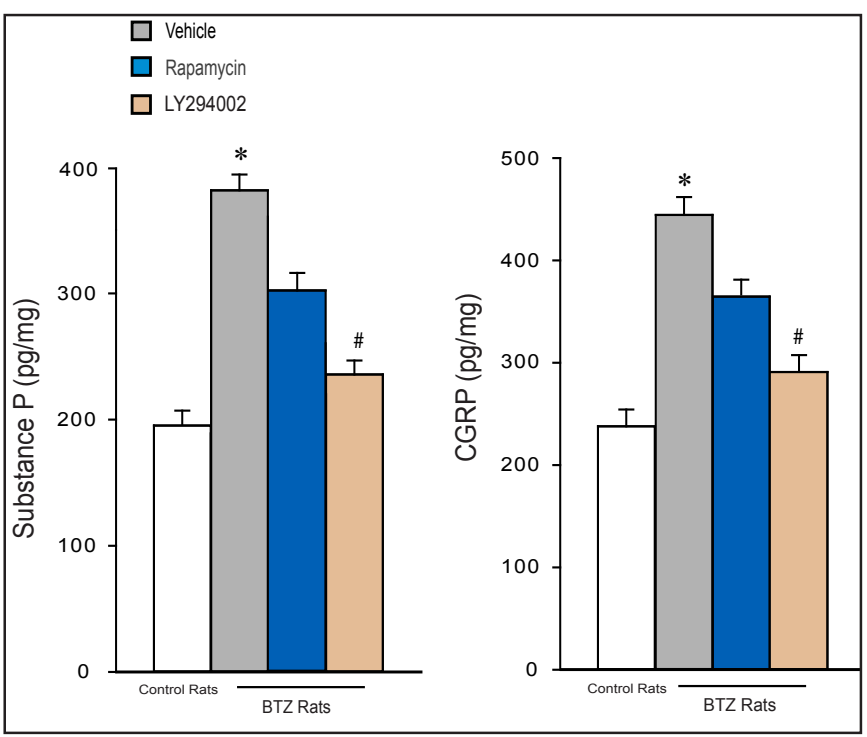




\section{Cellular Physiology Cell Physiol Biochem 2018;48:54-62 and Biochemistry Published online: July 11, $2018 \quad \begin{aligned} & \text { DOI: 10.1159/000491662 } 2018 \text { The Author(s). Published by S. Karger AG, Basel } \\ & \text { www.karger.com/cpb }\end{aligned}$ \\ Duan et al.: mTOR and Peripheral Neuropathic Pain}

of mTOR signal by intrathecal infusion of rapamycin alleviated mechanical pain and cold hypersensitivity. Inhibition of PI3K further attenuated mTOR expression and neuropathic pain.

We consistently observed development of mechanical hyperalgesia and cold hypersensitivity in BTZ rats. Results of the current study also demonstrated that $\mathrm{t}^{\mathrm{V}}$ expression of p-mTOR, mTOR-p-S6K1, p-4E-BP1 and p-PI3K pathway were upregulated BTZ rats as compared with control rats. Blocking mTOR by intrathecal infusion of rapamycin attenuated mechanical hyperalgesia and largely restored shortened time spent on the $c$ plate evoked by BTZ. Blocking PI3K signal attenuated expression of mTOR in PTZ rats also decreased mechanical and cold hypersensitivity. Moreover, enhanced spin abstar and CGRP evoked by BTZ were blunted after inhibition of either mTOR or PI3K.

The PI3K/Akt pathway is an intracellular signaling pathway in regula This important mechanism is directly related to cellular quiescence, longevity. PI3K can phosphorylate and activate Akt in the plasma mem ${ }^{-}$ leads to several downstream effects which alter transcription of p70 rit $\mathrm{BP} 1$ and activating cAMP response element-binding protein (CREB) a $[4,5,22,23]$. Our present study demonstrated that blocks $[4,5,22,23]$. Our present study demonstrated that block ${ }^{i}$ (3K atte $\mathrm{p}$-mTOR and p-S6K1 expression, and that intrathecal infusion of PI3K thenu. d hyperalgesia and restored shortened time spent on the cold plate obser in this suggests that $\mathrm{PI} 3 \mathrm{~K}$ is necessary to play a regulatory role in mediating the of IOR on BTZ-evoked mechanical pain and cold hypersensitivity res res.

It is well known that stimulation of nocicep eceptors is ensory nerves leads to the releases of substance $\mathrm{P}$ and CGRP from the rminal of dorsal root ganglions into the superficial dorsal horn $[18,24]$. Numerou cep vresent on the presynaptic site of the nerve terminals and contribute to the re s of wstance P and CGRP in regulating inflammatory pain [16]. It is assumed thathes can indirectly affect these receptors such as transient receptor ot 1 vanmloid 1 (TRPV1) and thereby decrease the levels of substance P and CGRP [ originated from the dorsal h er 25,26$]$. PI3K-mTOR signal pathway is likely to present in these interneur ntaining iwo neuropeptides. Thus, blocking PI3K-mTOR can decrease the levels of se ropeptides. Nevertheless, in our current study, the levels of substance P an $G R P$, as important neurotransmitters engaged in pain, were significantly increased e su arficial dorsal horn of BTZ rats. Moreover, the increased substance $\mathrm{P}$ and CCDP m cantly attenuated after respective injection of rapamycin and LY294002 ur ard suggest that amplified expression of spinal mTOR and its downstream $\mathrm{P}$ nY 1 or 4E-BP1 are engaged in BTZ-induced mechanical hyperalgesia and cold h rrse tiv mikely via the releases of substance P and CGRP.

$\mathrm{No}^{+}$we ved that LY294002 had a greater effect on substance P and CGRP th an rap in did, indicating the role played by PI3K as a upstream pathway in regulating sens sponses observed in BTZ rats via mTOR signals. This suggests that mTOR nly do nstream pathway of PI3K. Nevertheless, to the best of our knowledge there evidence specifically showing the role played by mTOR and PI3K in regulating
releases of spinal substance P and CGRP in a neuropathic pain model induced by BTZ. the present report suggest that substance P and CGRP regulated by mTOR and at the spinal level contribute to mechanical pain and cold hypersensitivity in BTZduced neuropathy.

In conclusion, in BTZ rats, spinal p-mTOR and mTOR-mediated p-S6K1 and p-4E-BP1 are upregulated, resulting in mechanical and cold hypersensitivity. Intrathecal administration of rapamycin has analgesic effects on BTZ-induced neuropathic pain. Also, blocking PI3K blunts the amplified expression of p-mTOR and thereby alleviates BTZ-evoked neuropathic pain. Nonetheless, our data for the first time reveal specific signal pathways leading to mechanical pain and cold hypersensitivity evoked by BTZ, including the activation of PI3K and MTOR downstream pathways, and releases of substance P and CGRP. Our data provide a base for the mechanisms responsible for BTZ-induced peripheral painful neuropathy. Targeting one

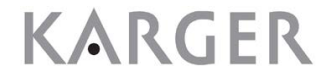




\section{Cellular Physiology Cell Physiol Biochem 2018;48:54-62 \begin{tabular}{c|c} 
DOI: 10.1159/000491662 & $\begin{array}{l}\text { O 2018 The Author(s). Published by S. Karger AG, Basel } \\
\text { www.karger.com/cpb }\end{array}$
\end{tabular} \\ Duan et al.: mTOR and Peripheral Neuropathic Pain}

or more of these signaling molecular mediators involved in activation of PI3K-mTOR at the spinal levels may present new opportunities for treatment and management of pain often observed in cancer patients during chemotherapeutic application of BTZ.

\section{Disclosure Statement}

No conflict of interests exists.

\section{References}

1 Adams J, Kauffman M: Development of the proteasome inhibitor Velcade (Bortezomib 2004;22:304-311.

2 Voorhees PM, Dees EC, O’Neil B, Orlowski RZ: The proteasome as a target for car $\urcorner$ e Res 2003;9:6316-6325.

-3 Curran MP, McKeage K: Bortezomib: a review of its use in patients 2009;69:859-888.

4 Hay N, Sonenberg N: Upstream and downstream of mTOR. Genes

5 Banko JL, Poulin F, Hou L, DeMaria CT, Sonenberg N, Klann E: The tra critical for eIF4F complex formation, synaptic plast and memory hippocampus. J Neurosci 2005;25:9581-9590.

6 Costa-Mattioli M, Sossin WS, Klann E, Sonenberg N: Tand memory. Neuron 2009;61:10-26.

7 Bao Y, Gao Y, Hou W, Yang L, Kong X, Zheng H, Li C, activated receptor 2 and mu-opioid recept 2015;137:1475-1483.

Geranton SM, Jimenez-Diaz L, Torsney C sensitive signaling pathway is 2009;29:15017-15027. in mice lacking the fragi 2007;27:13958-13967.

10 Xu Q, Fitzsimmon- R Ste Akt-mammal 2011;31:21 2

11 Asante Wal Vo s.ckenson AH: Formalin-induced behavioural hypersensitivity and neuronal hyp tabilit_ediated by rapid protein synthesis at the spinal level. Mol Pain 2009;5:27.

S, Wu X, Zhong J, Lv A, Jiao J, Chen Z: Blocking mammalian target of rapamycin alleviates bone ancer $d_{A} \quad d$ morphine tolerance via micro-opioid receptor. Int J Cancer 2016;138:2013-2020. rgado Q, erra PP, Tavares I: Neuronal hyperactivity at the spinal cord and periaqueductal grey during abetic neuropathy: effects of gabapentin. Eur J Pain 2010;14:693-699.

Silva IM, Amorim D, Almeida A, Tavares I, Pinto-Ribeiro F, Morgado C: Pronociceptive changes in the activity f rostroventromedial medulla (RVM) pain modulatory cells in the streptozotocin-diabetic rat. Brain Res Bull 2013;96:39-44.

Bouhassira D, Lantéri-Minet M, Attal N, Laurent B, Touboul C: Prevalence of chronic pain with neuropathic characteristics in the general population. Pain 2008;136:380-387.

16 Hua X-Y, Yaksh TL: Dorsal Horn Substance P and NK1 Receptors: Study of a Model System in Spinal Nociceptive Processing; in (Malcangio M, ed) Synaptic Plasticity in Pain. Springer Science Business Media, 2009, vol. p. pp.109-138.

17 Engel MA, Khalil M, Mueller-Tribbensee SM, Becker C, Neuhuber WL, Neurath MF, Reeh PW: The proximodistal aggravation of colitis depends on substance P released from TRPV1-expressing sensory neurons. J Gastroenterol 2012;47:256-265. 


\section{Cellular Physiology Cell Physiol Biochem 2018;48:54-62 \begin{tabular}{l|l} 
DOI: 10.1159/000491662 & $\begin{array}{l}\text { O } 2018 \text { The Author(s). Published by S. Karger AG, Basel } \\
\text { www.karger.com/cpb }\end{array}$
\end{tabular} \\ Duan et al.: mTOR and Peripheral Neuropathic Pain}

18 Lin Q, Li D, Xu X, Zou X, Fang L: Roles of TRPV1 and neuropeptidergic receptors in dorsal root reflexmediated neurogenic inflammation induced by intradermal injection of capsaicin. Mol Pain 2007;3:30.

19 Puttfarcken PS, Han P, Joshi SK, Neelands TR, Gauvin DM, Baker SJ, Lewis LG, Bianchi BR, Mikusa JP, Koenig JR, Perner RJ, Kort ME, Honore P, Faltynek CR, Kym PR, Reilly RM: A-995662 [(R)-8-(4-methyl-5-(4(trifluoromethyl)phenyl)oxazol-2-ylamino)-1, 2,3, 4-tetrahydr onaphthalen-2-ol], a novel, selective TRPV receptor antagonist, reduces spinal release of glutamate and CGRP in a rat knee joint pain model. Pain 2010;150:319-326.

20 Chaplan SR, Bach FW, Pogrel JW, Chung JM, Yaksh TL: Quantitative assessment of tactile allodynia in the paw. J Neurosci Methods 1994;53:55-63.

21 Wang D, Zhao J, Wang J, Li J, Yu S, Guo X: Deficiency of female sex hormones augments PGE within midbrain periaqueductal gray. J Neurol Sci 2014;346:107-111.

-22 Al-Batran SE, Ducreux M, Ohtsu A: mTOR as a therapeutic target in patients with gas 2012;130:491-496.

23 Shor B, Gibbons JJ, Abraham RT, Yu K: Targeting mTOR globally in cancer: thinking Cycle 2009;8:3831-3837.

24 Bevan S, Quallo T, Andersson DA: TRPV1. Handb Exp Pharmacol 2014;222:20

25 Chaouch A, Besson JM: [Peripheral and spinal mechanisms of noci $\eta$ ]. Rev Ne 1986;142:173-200.

26 Kozsurek M, Lukacsi E, Fekete C, Wittmann G, Rethelyi M, Puskar regulated transcript peptide (CART) is present in peptidergic $\mathrm{C}$ prin interneurons with a possible role in nociception in typerficial lam Neurosci 2007;26:1624-1631.
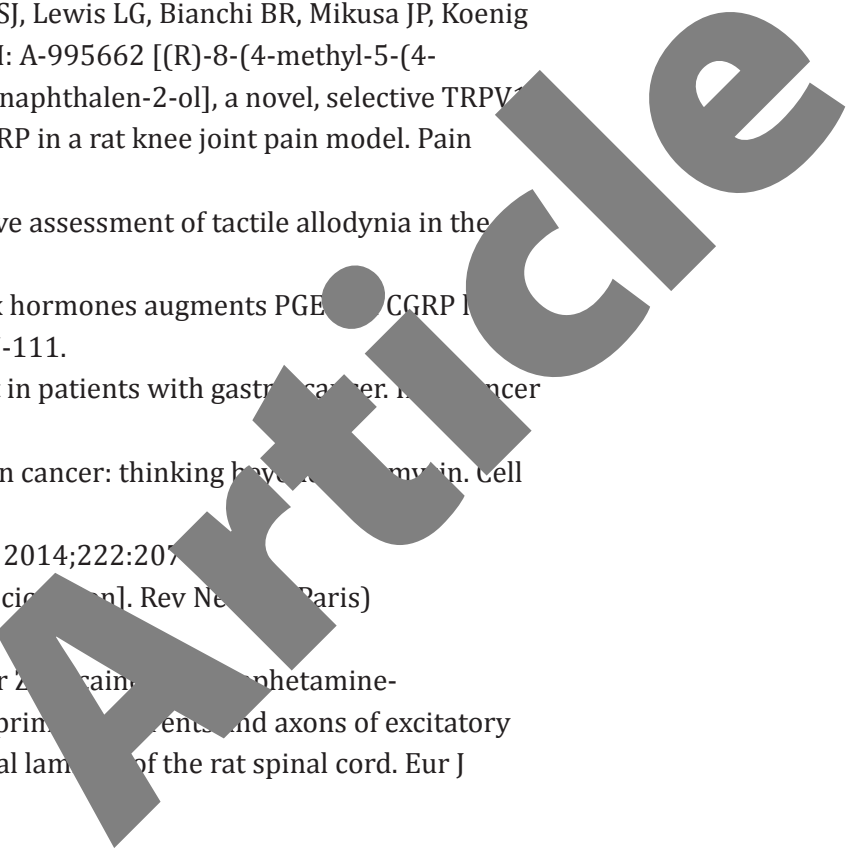
ent. nd axons of excitatory f the rat spinal cord. Eur J 